\title{
Virucidal Activity of Chlorine Dioxide Gas for Reduction of Coronavirus on Surfaces and PPE
}

\author{
Jeffrey Driver, George Lukasik, Marie Bourgeois, Patricia Tam, Raymond Harbison \\ Center for Environmental and Occupational Risk Analysis and Management, College of Public Health, University of South \\ Florida, Tampa, FL, USA \\ Email: rharbiso@usf.edu
}

How to cite this paper: Driver, J., Lukasik, G., Bourgeois, M., Tam, P. and Harbison, R. (2021) Virucidal Activity of Chlorine Dioxide Gas for Reduction of Coronavirus on Surfaces and PPE. Occupational Diseases and Environmental Medicine, 9, 13-19. https://doi.org/10.4236/odem.2021.91002

Received: January 8, 2021

Accepted: February 6, 2021

Published: February 9, 2021

Copyright (c) 2021 by author(s) and Scientific Research Publishing Inc. This work is licensed under the Creative Commons Attribution International License (CC BY 4.0).

http://creativecommons.org/licenses/by/4.0/

\begin{abstract}
A coronavirus (SARS-CoV-2) has caused a global pandemic and associated morbidity and mortality resultant from COVID-19. As a result of efforts to control direct (person to person) and indirect (contaminated objects, surfaces, indoor air) transmission of the virus, various interventions have been evaluated. Studies were conducted to evaluate the efficacy of commercially available chlorine dioxide (CD) products to reduce viral loads on PPE (face masks) and surfaces using a novel dry gas release intervention. The efficacy of CD slow release 30-day sachets was tested on N95 face masks inoculated with human coronavirus OC43 in suspension. One sachet was placed with an inoculated mask in plastic resealable bags. Three trials were completed using the original sachet where a mask and sachet were placed into a plastic bag for 13 hours per sachet age of 1 day, 14 days, and 30 days. The amount of CD generated during a 13-hour treatment period was $0.30 \mathrm{mg}$. The nominal concentration of CD was estimated to be $317 \mathrm{mg} / \mathrm{m}^{3}$. All three tests demonstrated at least a $99.91 \%$ reduction of viral loading in the mask versus a non-treated control. Efficacy of CD dry gas fast releasing pods (Ultrashok) for fumigation was also tested in a $1344 \mathrm{ft}^{3}$ closed room. Two pods were placed in the space and $\mathrm{CD}$ surface virucidal efficacy was tested in three locations of the room after 1 hour and 2 hours of dwell time. The estimated nominal peak concentration was $15 \mathrm{ppmv}$ in the room. The one-hour exposure saw a $>99.91 \%$ OC43 reduction on surfaces and the two-hour exposure resulted in a $>99.997 \%$ OC43 reduction on surfaces versus a non-treated control. These results indicate dry CD is highly effective against human coronavirus. CD was $99.91 \%$ effective for eliminating human coronavirus OC43 in both sachet and capsule fumigant form using both fast and slow release mechanisms. Rapid fumigant application is suitable for contaminated rooms, ambulances, emergency vehicles, and many types of PPE, most particularly porous PPE materials. The gaseous state of $\mathrm{CD}$ allows for rapid diffusion and transfer of the virucidal
\end{abstract}


stable free radical to all surfaces of PPE and indoor areas that would favor virus survival. Additionally, this work suggests $C D$ can be effective at levels with significant margins of safety (little to no exposure and rapid degradation of residuals) providing minimal public health risks associated with the use of CD.

\section{Keywords}

Chlorine Dioxide Gas, Coronavirus, Virucidal Activity

\section{Introduction}

Chlorine dioxide is currently approved by the United States Food and Drug Administration (FDA) and Environmental Protection Agency (EPA) for many antimicrobial uses, including water purification and food decontamination, thus increasing the shelf life of fruit, vegetables, and meat [1] [2]. CD is not a naturally occurring compound and is a gas at room temperature with a boiling point of $11^{\circ} \mathrm{C}$. Studies of human inhalation that were above the allowable concentration guideline have resulted in conjunctiva irritation, with no cerebral effects, and no carcinogenicity. The Occupational Safety and Health Administration (OSHA) and EPA both have the allowable time-weighted average exposure at 0.1 $\mathrm{ppm}$, with OSHA increasing the concentration to $0.3 \mathrm{ppm}$ for the short-term exposure limit. A study published by Akamatsu et al. in 2012 [3] found that rats exposed to $0.1 \mathrm{ppm}$ for 24 hours per day and 7 days per week for 6 months did not show CD gas-related toxicity, further demonstrating its safety.

December of 2019 saw the rise of a novel coronavirus (COVID-19) and has since grown into a global pandemic. As a result, the United States has seen shortages of personal protective equipment (PPE), leading to unnecessarily increased risk for people caring for COVID-19 positive patients like hospital workers and emergency medical technicians. CD's virucidal properties, lack of residue, and safety make it ideal for reusing various PPE such as N95 masks. This would also be applicable on a bigger scale when applied to surfaces in rooms that have been contaminated with the virus. Relatively short fumigation of enclosed spaces would allow for fast sterilization with less manual labor required of the cleaning staff.

Currently, liquid antimicrobial solutions like hydrogen peroxide and phenols are used to manually disinfect various spaces such as operating rooms and ambulances. CD's gaseous state allows it to reach and disinfect even the smallest crevices that may otherwise be ignored. $\mathrm{CD}$ is commercially sold across the country. One such company called ICA Tri-Nova, LLC produces delayed-release $\mathrm{CD}$ products that range from 1 hour to 30 days, to be used for water purification, odor reduction, and more. One of its products is the StayFresh UltraShok, which is a small pod that quickly releases $\mathrm{CD}$ gas for up to 1 hour. The purpose of this study was to evaluate the virucidal efficacy of this product for reducing 
coronavirus contaminated facemasks and surfaces.

\section{Methods}

\subsection{Sachet Use on N95 Facemasks}

The test material was contained in a sachet shown in Figure 1. The materials consisted of a slow releasing chlorine dioxide reagent each in a clear sealing $\mathrm{PE}$ plastic bag. The study was performed to evaluate the virucidal efficacy against human coronavirus OC43 on inoculated facemasks. The study protocol was an adaptation from ASTM E1053 [4]. Briefly, one hundred microliters of virus suspension were added to each of marked sections on three N95 facemasks. The inoculation was added as a thin layer. Once inoculated, each of 2 masks was placed in a sealed humidified plastic nylon clean bag with a chlorine dioxide sachet. One mask was added to each of the bags. The third facemask was added to a humidified bag not containing the slow release sachet. This served as the recovery control. A NIST traceable laboratory timer was started. Following overnight contact time ( $14 \pm 1$ hours), each of the facemasks was removed from the respective bags and the marked sections were aseptically cut. The sections were each added to sterile containers with $10 \mathrm{~mL}$ of D/E Neutralizing Broth (Criterion) and were homogenized. The samples were analyzed on the day of collection at undiluted and at ten-fold dilutions in replicates of 5 for viable infectious coronavirus. The entire study was repeated again following 2 weeks using the same bags and sachets and again following 30 days after start of exposure. Positive, negative and neutralization controls were used to provide quality control and reference data as per laboratory standard accredited ISO17025:2017 methodology.

\subsection{Canister Use on Room Surfaces}

Surface virucidal efficacy was tested using plastic capsules shown in Figure 2 containing a powder that required activation to release chlorine dioxide gas. The study was performed to evaluate virucidal efficacy against human Coronavirus OC43 on inoculated carriers placed at random locations within an enclosed

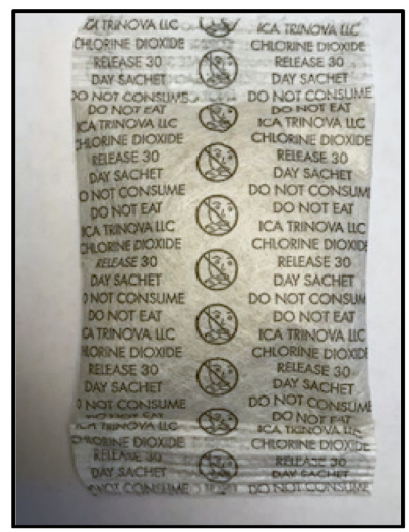

Figure 1. CD sachet. 


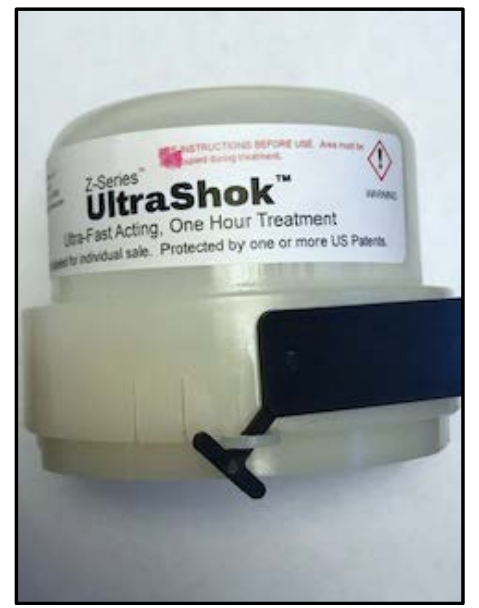

Figure 2. CD canister.

room. The size of the room was $1344 \mathrm{ft}^{3}$ (approximately $7^{\prime} \times 24^{\prime} \times 8^{\prime}$ ). The study was conducted as per laboratory protocol adaptation from ASTM E1053 [4]. Briefly, one hundred microliters of virus suspension (containing $5 \%$ heat inactivated Fetal Bovine Serum) was added as a thin layer to each $100 \mathrm{~mm}$ glass petri dish and allowed to dry. The carriers were placed at random locations in the room:

Three carriers were placed at locations open to room air. Two of the capsules were placed on the floor in the room and spaced at equal length intervals. The temperature of the room was maintained at $19-21$ degrees $C$. This resulted in low relative humidity $(\mathrm{RH})$. An ultrasonic humidifier was placed into the room to maintain humidity at $75 \%-85 \%$ RH. Additionally, a small 510 CFM fan was also placed in the room to allow air circulation and homogeneity of conditions in the room. The capsules were activated and immediately the room was vacated and sealed. The room was allowed a dwell time of 1 and 2 hours.

The room was opened, a mild chlorine-based odor was noted but it was not overwhelming or irritating. Each of the carriers was removed, immediately added to sterile containers with $10 \mathrm{~mL}$ of $\mathrm{D} / \mathrm{E}$ Neutralizing Broth, and homogenized. The samples were analyzed for viable infectious coronavirus OC43 on the day of the study at undiluted and at ten-fold dilutions in replicates of five. Additionally, two control glass carriers were inoculated as above but maintained in a separate area not exposed to the gas. They were processed similarly following recovery of treatment carriers. These served as recovery controls. The number of microorganisms recovered from the controls was used to calculate the starting concentration. Positive, negative and neutralization controls were included with test materials to provide quality control and reference data as per laboratory standard accredited ISO17025:2017 methodology [5].

Viable virus was analyzed using HRT-18G cell infectivity assay. Cell monolayers were monitored for cytopathic effect development over a 14-day period. Viruses were enumerated as Infectious Units (I.U.) using the Most Probably Number (MPN) analysis of the cell culture results. Analysis was conducted as 
per method EPA/600/R-95/178 [6] and reported as I.U./Carrier section. All equipment and supplies were validated to or were calibrated to NIST traceable standards. All QC were within method acceptance limit. No general environmental conditions are specified in the standard or have been identified that could affect the test results or measurements.

\section{Results and Discussion}

Gas releasing sachets effectively decontaminated N95 facemasks. Facemasks contaminated with human coronavirus OC43 and exposed to chlorine dioxide gas for 13 hours were decontaminated with a $99.9 \%$ reduction of the virus in the facemasks, shown in Figure 3. Infectious virus was not detected in the facemask samples analyzed. Similar results were observed following a 14-day and 30-day re-challenge, shown in Figure 4 and Figure 5, respectively.

Gas releasing plastic capsules effectively eliminated virus contaminated surfaces. Surfaces contaminated with virus were placed at various locations in an enclosed room and subsequently exposed to chlorine dioxide gas. Chlorine dioxide gas exposure eliminated $99.99 \%$ of the surface virus. Infectious virus was not detected on contaminated surfaces at 1 and 2 hours following chlorine dioxide fumigation in Figure 6 and Figure 7, respectively.

Chlorine dioxide was greater than $99.9 \%$ effective at eliminating human coronavirus OC43 contamination in facemasks and on room surfaces.

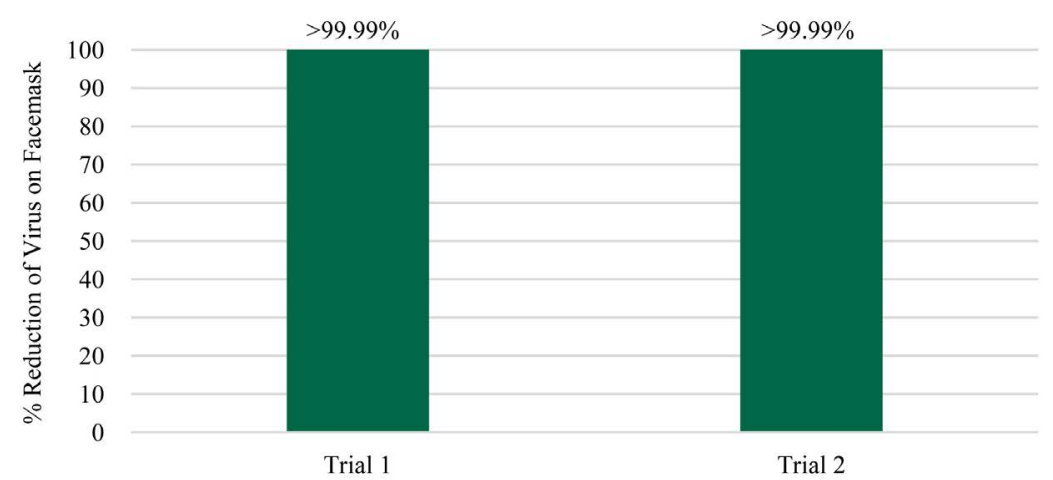

Figure 3. Facemask contaminated with coronavirus OC43. 13-hour contact with CD.

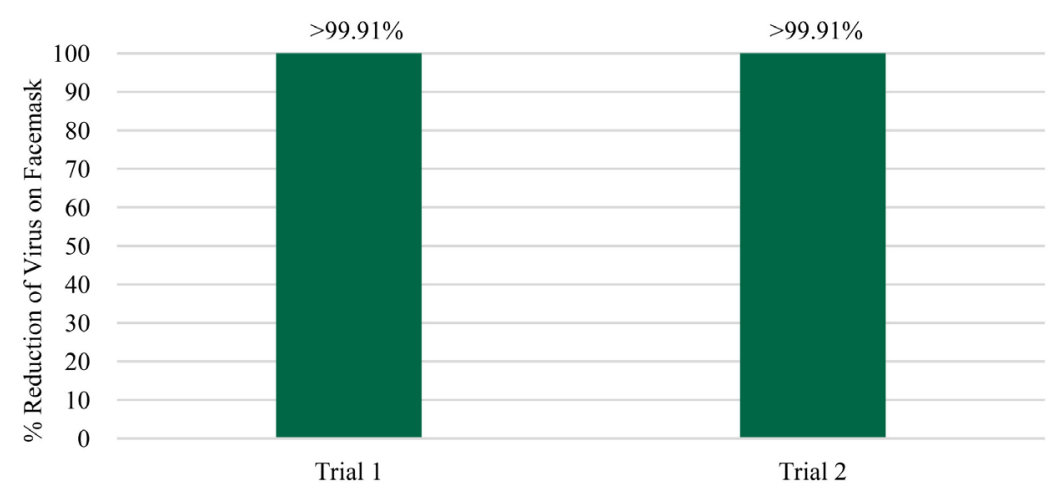

Figure 4. Facemask contaminated with coronavirus OC43. 13-hr 14-day re-challenge. 


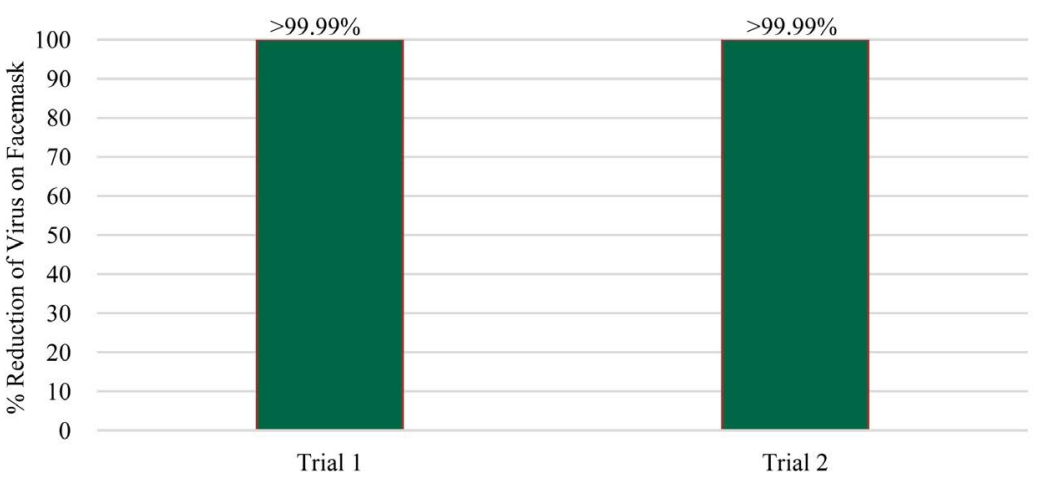

Figure 5. Facemask contaminated with coronavirus OC43. 13-hr 30-day re-challenge.

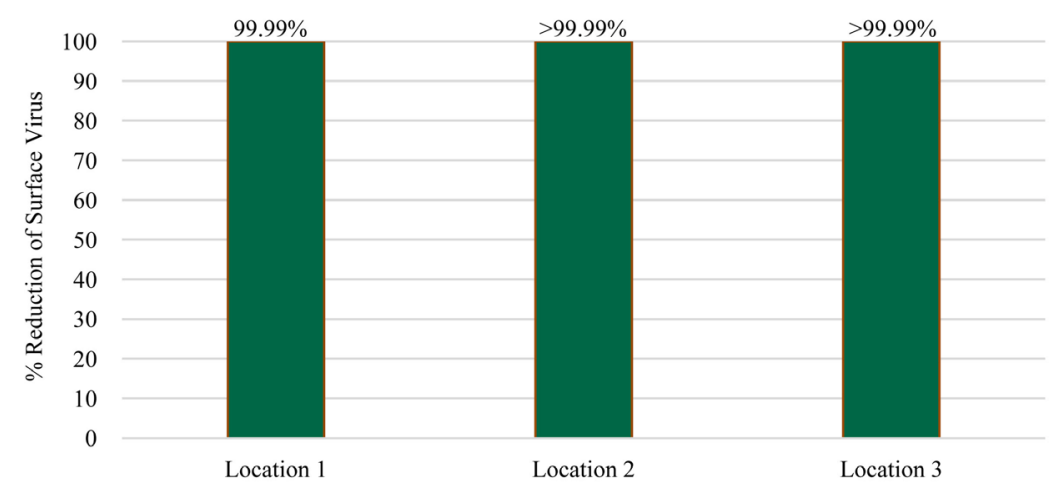

Figure 6. Room surfaces contaminated with human coronavirus OC43. 1-hour contact with chlorine dioxide.

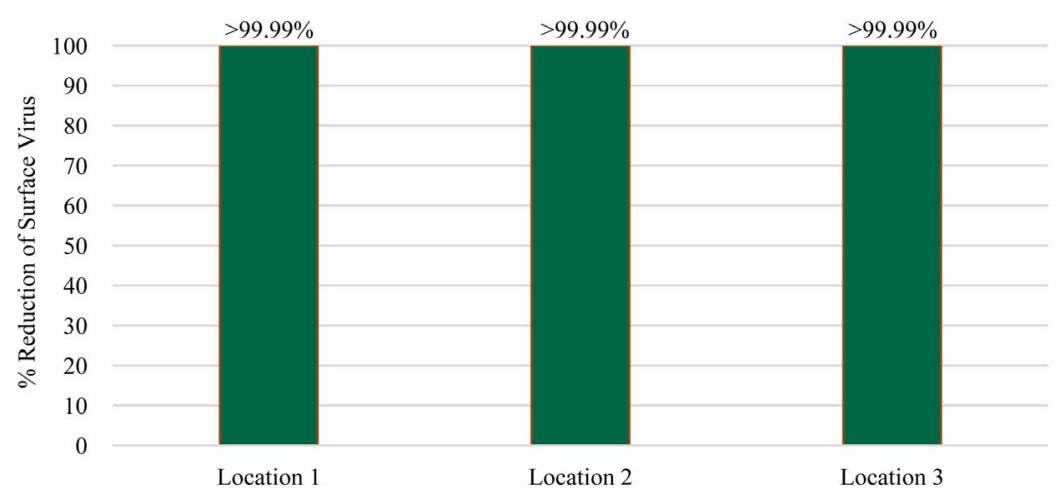

Figure 7. Room surfaces contaminated with human coronavirus OC43. 2-hour contact with chlorine dioxide.

\section{Conclusion}

Chlorine dioxide gas was reported as effective at preventing aerosol-induced influenza virus infection [7]. Gas releasing sachets effectively decontaminated N95 facemasks. Gas releasing plastic capsules effectively eliminated virus contaminated surfaces. Chlorine dioxide was greater than $99.9 \%$ effective at eliminating human coronavirus OC43 contamination in facemasks and on room surfaces. Rapid fumigant application is suitable for contaminated rooms, ambulances, emergency vehicles, and many types of PPE materials. This work demonstrates 
chlorine dioxide gas can be effective at levels with significant margins of safety (little to no exposure and rapid degradation of residuals) providing minimal public health risks associated with the use of chlorine dioxide gas.

\section{Acknowledgements}

Funding for laboratory testing at BCS Laboratories was provided by ICA Tri-Nova, LLC.

\section{Conflicts of Interest}

The authors declare no conflicts of interest regarding the publication of this paper.

\section{References}

[1] NIH (National Institutes of Health) (2021) National Library of Medicine. https://pubchem.ncbi.nlm.nih.gov/compound/Chlorine-dioxide

[2] U.S. EPA (Environmental Protection Agency) (2006) Reregistration Eligibility Decision (RED) for Chlorine Dioxide and Sodium Chlorite (Case 4023). EPA 738-R-06-007. Washington DC.

[3] Akamatsu, A., et al. (2012) Six-Month Low Level Chlorine Dioxide Gas Inhalation Toxicity Study with Two-Week Recovery Period in Rats. Journal of Occupational Medicine and Toxicology, 7, 2. https://doi.org/10.1186/1745-6673-7-2

[4] ASTM (2011) E1053-11. Standard Test Method to Assess Virucidal Activity of Chemicals Intended for Disinfection of Inanimate, Nonporous Environmental Surfaces. ASTM International, West Conshohocken, PA.

[5] ISO/CASCO Committee on Conformity Assessment (2017) General Requirements for the Competence of Testing and Calibration Laboratories. ISO/IEC 17025:2017. Corrected Version: 2018-03. International Organization for Standardization. Geneva, Switzerland.

[6] U.S. EPA (Environmental Protection Agency) (1996) ICR Microbial Laboratory Manual. EPA/600/R-95/178. Office of Research and Development, Washington DC.

[7] Ogata, N. and Shibata. T. (2008) Protective Effect of Low Concentration Chlorine Dioxide Gas against Influenza A Virus Infections. Journal of General Virology, 89, 60-67. https://doi.org/10.1099/vir.0.83393-0 